\title{
Laparoscopic Excision of Anterior Abdominal Wall Tumors: A Case of Desmoid-Type Fibromatosis Arising in the Rectus Muscle
}

\author{
Ho Jun Lee ${ }^{1}$, Hye-Jeong Jeong, M.D. ${ }^{2}$, Jin Woo Lee, M.D. ${ }^{2}$, Sung Hoon Choi, M.D. ${ }^{2}$ \\ ${ }^{1}$ CHA University, Seongnam, ${ }^{2}$ Department of Surgery, CHA Bundang Medical Center, CHA University, Seongnam, Korea
}

Purpose: Laparoscopic approaches to the anterior abdominal wall are difficult because of the inherent limitations of laparoscopic surgery.

Methods: A 25-year-old young female visited to our hospital with an incidentally-found palpable abdominal mass. Computed tomography revealed a $7-\mathrm{cm}$ round enhancing mass arising from the left posterior rectus sheath. The patient underwent laparoscopic excision of the inner mass arising from the anterior abdominal wall.

Results: The operation lasted for 45 minutes and there was no measurable bleeding during the procedure. Pathological assessment revealed that the tumor was a DTF $7.0 \mathrm{~cm}$ in size. The patient was discharged on the second postoperative day in good condition, and is currently being followed on a routine basis for surveillance without adjuvant therapy.

Conclusion: Laparoscopic approach for the anterior abdominal wall tumor could be feasible in select patients. This minimally-invasive approach helps to ensure good cosmetic outcomes and quality of life.

Keywords: Laparoscopy, Minimally invasive, Abdominal fibromatosis, Abdominal wall
Received July 25, 2017

Revised August 23, 2017

Accepted September 5, 2017

Corresponding author

Sung Hoon Choi

Department of Surgery, CHA

Bundang Medical Center, CHA

University, Caner Research Building \#524, 59 Yatap-ro, Bundang-gu,

Seongnam 13496, Korea

Tel: +82-31-780-2990

Fax: +82-31-780-1824

E-mail: feel415@cha.ac.kr

ORCID:

http://orcid.org/0000-0002-1664-3727

Supplementary video file: This article contains supplementary material (https://doi.org/10.7602/ jmis.2018.21.1.46).

This is an Open Access article distributed under the terms of the Creative Commons Attribution Non-Commercial License (http:// creativecommons.org/licenses/by-nc/4.0/) which permits unrestricted non-commercial use, distribution, and reproduction in any medium, provided the original work is properly cited.

Copyright $@ 2018$ The Journal of Minimally Invasive Surgery. All rights reserved.

\section{INTRODUCTION}

Benign fibrous tumors originating from the musculature and fascial structures frequently involve the rectus sheath $(64 \%)^{1,2}$ Wide excision with clear margins is the gold-standard treatment option. ${ }^{3,4}$ Surgical excision is generally performed using an open laparotomy approach resulting in a large postoperative scar despite the benign characteristics of the tumor.

Laparoscopic approaches to the anterior abdominal wall are difficult because of the inherent limitations of laparoscopic surgery, i.e., the limited range of motion of straight instru- ments without joints and movement via the fulcrum effect. ${ }^{5}$ We describe the successful laparoscopic excision of a desmoidtype fibromatosis arising from the posterior rectus sheath in a young woman with no operative or trauma history.

\section{MATERIALS AND METHODS}

A 25-year-old young female visited our hospital with an incidentally-detected palpable abdominal mass. The mass was hard, round, and unfixed, but its deep location suggested that it originated from either the abdominal wall or intra-abdom- 


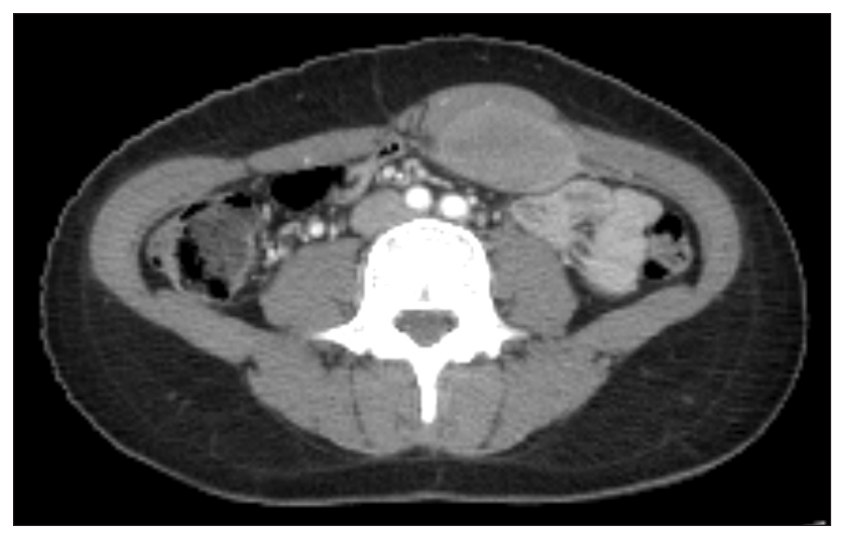

Fig. 1. Computed tomography (CT) revealed an 8-cm oval-shaped enhancing mass arising from the left posterior rectus sheath at the umbilical level and involving the rectus muscle.

inal organs. Computed tomography (CT) revealed an $8-\mathrm{cm}$ oval-shaped enhancing mass arising from the left posterior rectus sheath at the umbilical level and involving the rectus muscle (Fig. 1). The CT findings suggested fibromatosis, with a differential diagnosis of well-differentiated sarcoma. The patient was young and unmarried; therefore, we decided to use a laparoscopic approach.

The patient's body mass index (BMI) was $22.41 \mathrm{~kg} / \mathrm{m}^{2}$. A $12-\mathrm{mm}$ camera port was introduced at the right flank and two 5-mm working trocars were added at the right upper quadrant and the right suprapubic area to allow straight laparoscopic instruments to approach the tumor in the oblique direction (Fig. 2). A round bulging mass was identified on the peritoneum. The resection margin was demarcated around the tumor with electrocautery and an incision was made including the posterior rectus sheath. The tumor was then retracted using the incised sheath. The tumor involved the rectus muscle, which was resected using a Harmonic scalpel (Ethicon, Sommerville, NJ, USA). The tumor was completely removed and the focal defect of the fascia was approximated with $\mathrm{V}-\mathrm{Loc}^{\mathrm{TM}}$ (Covidien Medtronic, St. Paul, MN, USA) by intracorporeal suturing. The specimen was retrieved using a plastic bag through a small extension of the lower 5-mm trocar site, which was hidden by inner wear (Fig. 2). An indwelling drain tube was not placed.

\section{RESULTS}

The operation lasted for 45 minutes and there was no measurable bleeding during the procedure. The largest wound was only about $2.5 \mathrm{~cm}$. Pathological assessment revealed that the tumor was a desmoid-type fibromatosis $8.0 \mathrm{~cm}$ in size. The tumor was completely removed without fragmentation, but it

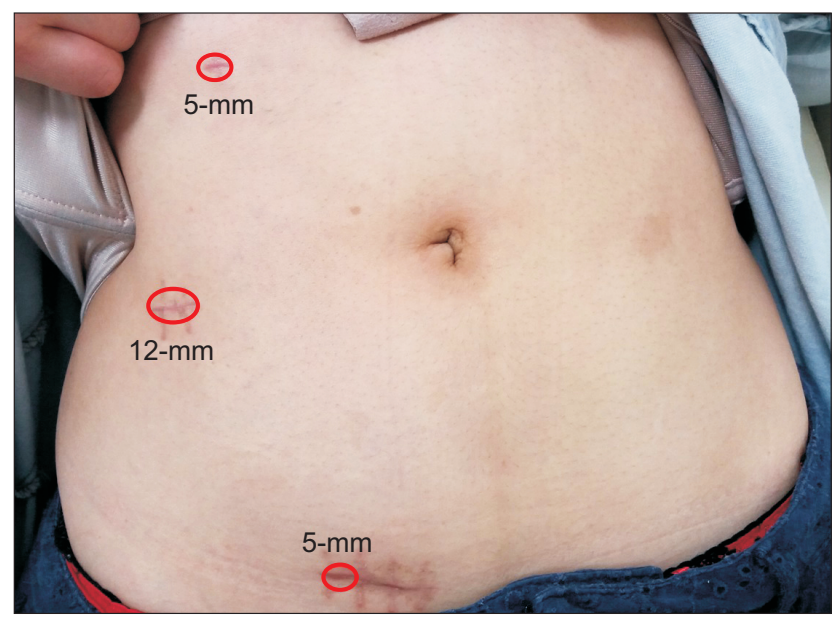

Fig. 2. A 12-mm camera port was introduced at the right flank and two 5-mm working trocars were added at the right upper quadrant and the right suprapubic area to allow straight laparoscopic instruments to approach the tumor in the oblique direction.

was focally exposed through the surgical margin on the rectus muscle. Postoperative recovery was uneventful and the patient was discharged on the second postoperative day in good condition. She is currently being followed on a routine basis without adjuvant therapy.

\section{DISCUSSION}

Fibromatosis are benign soft-tissue tumors and they are classified as superficial or deep according to location. ${ }^{6,7}$ Superficial-type fibromatosis commonly arise at palmar, plantar, and penile locations, ${ }^{7}$ while deep fibromatosis occur in the deep extra-abdomen, abdomen, and intra-abdomen, which also represent subclassifcations. ${ }^{2,8}$ These deep types are called desmoid-type fibromatosis, and are defined as clonal fibroblastic proliferations arising in the deep soft tissue by the World Health Organization. ${ }^{8}$ Therefore, desmoid-type fibromatosis (DTFs) can occur in any part of body with musculoaponeurotic structure. The present case describes an abdominal DTF. Abdominal DTFs commonly arise from the rectus or internal oblique muscle or aponeurosis in young women. For this reason, open surgical excision has been the common operative strategy, because the straight laparoscopic camera and instrument have inherent limitations in reaching and manipulating lesions located on the same side. To overcome such drawbacks, we inserted the trocars on the opposite side from the lesion and as laterally as possible from the center of abdomen. Tumor traction was facilitated by initial excision of the surrounding peritoneum and fascia. With these techniques, we successfully removed an anterior abdominal wall tumor and 
reconstructed the fascial defect.

The etiology of DTF has been suggested to be multifactorial, including germline mutations, hormones, trauma, and sporadic. ${ }^{1.9}$ Our case was a sporadic occurrence without any specific risk factors such as physical trauma or genetic disorders such as familial adenomatous polyposis syndrome or Gardner syndrome. The mainstay of treatment for sporadic DTF is complete surgical resection. Even when clear surgical margins are achieved, the local recurrence rate is quite high $(8 \sim 31 \%)^{3,10}$ Salas et al. evaluated the risk factors associated with sporadic DTF in a retrospective review, which suggested that age $<37$ years old, tumor size $>7 \mathrm{~cm}$, and extra-abdominal type were high risk of recurrence. ${ }^{11}$ Adjuvant options including radiotherapy and systemic therapies have been administered in patients with a poor prognosis; however, evidence regarding the efficacy of adjuvant therapy is sparse. ${ }^{3}$

While DTFs are benign neoplasms, large skin incisions are required for wide excision to minimize local recurrence. A laparoscopic approach to abdominal-type DTFs arising in the anterior abdominal wall and protruding into the abdominal cavity could be feasible in select patients. This minimally invasive approach improves cosmetic outcomes and quality of life, especially in young women.

\section{REFERENCES}

1) Kumar V, Khanna S, Khanna AK, Khanna R. Desmoid tumors: experience of 32 cases and review of the literature. Indian J Cancer 2009;46:34-39.

2) Wang Y, Cui NY, Li L, et al. An abdominal desmoid-type fibro- matosis. Quant Imaging Med Surg 2013;3:228-230.

3) Howard JH, Pollock RE. Intra-Abdominal and Abdominal Wall Desmoid Fibromatosis. Oncol Ther 2016;4:57-72.

4) Huang K, Wang CM, Chen JG, et al. Prognostic factors influencing event-free survival and treatments in desmoid-type fibromatosis: analysis from a large institution. Am J Surg 2014;207:847854.

5) Jang JY, Choi SH, Hwang HK, Kang CM, Lee WJ. Single-fulcrum Laparoscopic Cholecystectomy: A Variant Type of Single Incision and Multiport Technique. J Minim Invasive Surg 2011;14:51-55.

6) Walker EA, Petscavage JM, Brian PL, Logie CI, Montini KM, Murphey MD. Imaging features of superficial and deep fibromatoses in the adult population. Sarcoma 2012;2012:215810.

7) Montgomery E, Lee JH, Abraham SC, Wu TT. Superficial fibromatoses are genetically distinct from deep fibromatoses. Mod Pathol 2001;14:695-701.

8) Kasper B, Strobel P, Hohenberger P. Desmoid tumors: clinical features and treatment options for advanced disease. Oncologist 2011;16:682-693.

9) Bertario L, Russo A, Radice P, et al. Genotype and phenotype factors as determinants for rectal stump cancer in patients with familial adenomatous polyposis. Hereditary Colorectal Tumors Registry. Ann Surg 2000;231:538-543.

10) Peng PD, Hyder O, Mavros MN, et al. Management and recurrence patterns of desmoids tumors: a multi-institutional analysis of 211 patients. Ann Surg Oncol 2012;19:4036-4042.

11) Salas S, Brulard C, Terrier P, et al. Gene Expression Profiling of Desmoid Tumors by cDNA Microarrays and Correlation with Progression-Free Survival. Clin Cancer Res 2015;21:4194-4200. 